\title{
Non-additive QTL mapping of lactation traits in 124,000 cattle reveals novel recessive loci
}

\author{
Edwardo G. M. Reynolds ${ }^{1 *}$ (1) , Thomas Lopdell ${ }^{2}$, Yu Wang ${ }^{2}$, Kathryn M. Tiplady ${ }^{1,2}$, Chad S. Harland², \\ Thomas J. J. Johnson ${ }^{2}$, Catherine Neeley ${ }^{2}$, Katie Carnie ${ }^{2}$, Richard G. Sherlock ${ }^{2}$, Christine Couldrey ${ }^{2}$, \\ Stephen R. Davis², Bevin L. Harris², Richard J. Spelman², Dorian J. Garrick and Mathew D. Littlejohn 1,2
}

\begin{abstract}
Background: Deleterious recessive conditions have been primarily studied in the context of Mendelian diseases. Recently, several deleterious recessive mutations with large effects were discovered via non-additive genome-wide association studies (GWAS) of quantitative growth and developmental traits in cattle, which showed that quantitative traits can be used as proxies of genetic disorders when such traits are indicative of whole-animal health status. We reasoned that lactation traits in cattle might also reflect genetic disorders, given the increased energy demands of lactation and the substantial stresses imposed on the animal. In this study, we screened more than 124,000 cows for recessive effects based on lactation traits.
\end{abstract}

Results: We discovered five novel quantitative trait loci (QTL) that are associated with large recessive impacts on three milk yield traits, with these loci presenting missense variants in the DOCK8, IL4R, KIAA0556, and SLC25A4 genes or premature stop variants in the ITGAL, LRCH4, and RBM34 genes, as candidate causal mutations. For two milk composition traits, we identified several previously reported additive QTL that display small dominance effects. By contrasting results from milk yield and milk composition phenotypes, we note differing genetic architectures. Compared to milk composition phenotypes, milk yield phenotypes had lower heritabilities and were associated with fewer additive QTL but had a higher non-additive genetic variance and were associated with a higher proportion of loci exhibiting dominance.

Conclusions: We identified large-effect recessive QTL which are segregating at surprisingly high frequencies in cattle. We speculate that the differences in genetic architecture between milk yield and milk composition phenotypes derive from underlying dissimilarities in the cellular and molecular representation of these traits, with yield phenotypes acting as a better proxy of underlying biological disorders through presentation of a larger number of major recessive impacts.

\section{Background}

Non-additive genetic effects are best known from studies of Mendelian diseases, where recessive conditions have been shown to have major deleterious impacts on health and performance. These studies have mostly used

*Correspondence: edwardo.reynolds.1@uni.massey.ac.nz; egmreynolds@gmail.com

${ }^{1}$ Massey University, Palmerston North, New Zealand

Full list of author information is available at the end of the article a 'forward genetics' approach, where the observation of a disease phenotype precedes fine mapping and sequencing to highlight the mutation [1-3]. However, the reverse approach has also been applied, which first identifies candidate loss-of-function genotypes and subsequently performs phenotyping on traits likely to reflect the impact of the mutation [4-6]. Genome-wide association studies (GWAS) have been used to investigate non-additive effects in quantitative traits, but the number of findings remains limited in comparison to additive effects, where original author(s) and the source, provide a link to the Creative Commons licence, and indicate if changes were made. The images or other third party material in this article are included in the article's Creative Commons licence, unless indicated otherwise in a credit line to the material. If material is not included in the article's Creative Commons licence and your intended use is not permitted by statutory regulation or exceeds the permitted use, you will need to obtain permission directly from the copyright holder. To view a copy of this licence, visit http://creativecommons.org/licenses/by/4.0/. The Creative Commons Public Domain Dedication waiver (http://creativeco mmons.org/publicdomain/zero/1.0/) applies to the data made available in this article, unless otherwise stated in a credit line to the data. 
most such analyses fit an additive model only. Recent studies of non-additive effects include the investigation of complex traits in both humans [7] and cattle [8-12]. In cattle, Reynolds et al. [12] identified several recessive mutations with major negative impacts on growth and developmental traits, where some of these effects were found to be due to underlying genetic syndromes.

The concept of using routinely gathered, quantitative traits as proxies of genetic disorders is based on the idea that phenotypes such as growth or liveweight might be indicative of the overall health status of the animal, e.g. reduced growth could be caused by an underlying genetic disorder, in which case such effects could be detected via GWAS. Thus, it is relevant to investigate whether other easily measured traits might also serve as proxies of animal fitness, with a view to extend the scope of this approach. Lactation traits such as milk volume comprise one of the most commonly targeted classes of quantitative traits studied in cattle, where additive analyses of these traits have identified numerous candidate causative genes such as DGAT1 [13], GHR [14], ABCG2 [15], GPAT4 [16], and MGST1 [17]. Lactation traits might also reflect genetic disorders, given the increased energy demands of lactation and the substantial metabolic and physiological stresses imposed on the animal [18]. Thus, we were interested in investigating whether the application of non-additive models to lactation data might identify recessive mutations in addition to those found for growth traits, and to this end, have conducted non-additive GWAS for milk traits on 124,000 animals. We contrast the additive and non-additive genetic architectures of milk yield traits and milk composition traits. Finally, we describe the discovery of several novel major effect recessive loci and highlight candidate mutations that could underlie these undiagnosed recessive disorders.

\section{Methods}

\section{Animal populations}

The dataset reported in this study consists of 124,364 New Zealand dairy cattle. These animals come from a mixed breed population, where 20,893 were recorded as $16 / 16^{\text {th }}$ ' Holstein-Friesian (HF), 13,184 were recorded as

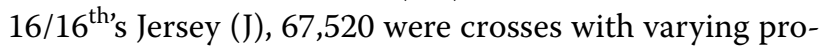
portions of the two breeds (HFXJ), and 22,767 were HF or J crossbreeds with minor proportions of other breeds including Ayrshire, Brown Swiss, or Hereford (and other crosses). The breed of an individual may be coded as $16 / 16^{\text {th }} \mathrm{s}$, however, this does not preclude the possibility that an ancestor may have been crossbred since matings between $15 / 16^{\text {th }} \mathrm{s}$ and $16 / 16^{\text {th }} \mathrm{s}$ animals are recorded as producing $16 / 16^{\text {th }} \mathrm{s}$ offspring. The animals were born between 1990 and 2018 with a mean birth year of 2010 .

\section{Phenotypes}

We analysed five first-lactation yield deviation phenotypes: three milk yield traits, i.e. milk volume (L/ lactation; a lactation refers to a standardised 268-day lactation period; $\mathrm{N}=124,356)$, milk protein yield $(\mathrm{kg} /$ lactation; $\mathrm{N}=124,356$ ), and milk fat yield ( $\mathrm{kg} /$ lactation; $\mathrm{N}=124,356$ ); and two milk composition traits, i.e. milk protein percentage $(\% ; \mathrm{N}=124,363)$, and milk fat percentage (\%; $N=124,363)$. Milk protein yield and milk fat yield are calculated on individual herd tests and are the product of the herd test milk volume multiplied by the herd test milk protein percentage or milk fat percentage, respectively.

Prior to genetic analysis, the phenotypes were adjusted for non-genetic effects obtained from the national genetic evaluation of the entire cattle population $\left(30 \times 10^{6}\right.$ animals $)$, which fits a mixed linear model, including effects for: contemporary group, age at calving, stage of lactation, and record type (i.e. am milkings, or pm milkings, or both). Since the number of herd-test measurements varies for each animal, these adjusted test day phenotypes were aggregated to a first lactation phenotypic deviation such that each animal has a single record and a corresponding weighting that reflects the amount of information contributing to the record [19].

\section{Reference population for sequence-based imputation}

Whole-genome sequencing was performed on 1300 animals that were mostly ancestral sires and represented the reference population for sequence-based imputation. These animals: HF $(\mathrm{N}=306), \mathrm{J}(\mathrm{N}=219)$, HFXJ $(\mathrm{N}=717)$, or other breeds and crossbreeds $(\mathrm{N}=58)$; were sequenced on Illumina HiSeq 2000 instruments targeting 100-bp paired-end reads. The sequence data were aligned to the ARS-UCD1.2 reference genome assembly using the Burrows-Wheeler alignment algorithm (BWA) version 0.7.17 [20], which resulted in a mean read depth of $15 \times$. For variant calling, we used the Genome Analysis ToolKit (GATK) v4.0.6.0 [21], followed by filtering of the variants with the variant quality score recalibration technique [21]. Based on the animals with a read depth $>10 \times(\mathrm{N}=850)$, variants that were singletons or were multi-allelic, had a map quality score lower than 50 , or a Mendelian error rate higher than $5 \%$, were filtered out leaving $21,005,869$ whole-genome sequence variants. The genotypes at the positions of these filtered variants were extracted from the sequence data of all 1300 animals and were phased using the software Beagle 5.0 [22] to generate the sequence-based imputation reference panel. 


\section{Genotyping}

DNA was extracted either from ear-punch tissue samples or blood samples for the 124,364 animals included in our study. These samples were processed to extract DNA at GeneMark (Hamilton, New Zealand) using Qiagen BioSprint kits, or at GeneSeek (Lincoln, NE, USA) using the Life Technologies' MagMAX system. Genotyping was performed using a variety of single nucleotide polymorphism (SNP) arrays including GeneSeek GGPv1 (8729 SNPs), GGPv2 (20,012 SNPs), GGPv2.1 (20,015 SNPs), GGPv3 (31,813 SNPs), GGPv3.1 (31,945 SNPs), GGPv4 (37,092 SNPs), GGP50kv1 (48,156 SNPs), GGP50kv1.1 (48,161 SNPs), Illumina BovineSNP50v1 (53,126 SNPs), Illumina BovineSNP50v2 (53,629 SNPs), or the BovineHD (772,235 SNPs) chips.

Consolidation of SNP-chip panels for sequence imputation Imputation from the genotyping panels to sequence resolution was performed as described in Wang et al. [23]. The various genotyping panels were grouped into four sets: GGP panels (GGPv1, GGPv2, GGPv2.1, GGPv3, GGPv3.1, and GGPv4), 50K panels (BovineSNP50v1 and BovineSNP50v2), GGP50k panels (GGP50kv1 and GGP50kv1.1), and the BovineHD panel. Animals genotyped on the GGP panels were imputed to the BovineSNP50v1 panel, then combined with the physically genotyped $50 \mathrm{~K}$ panel animals and successively imputed to the BovineHD panel. Animals genotyped on the GGP50k panels were separately imputed to the BovineHD panel in a single step. In order to incorporate the custom content that had been genotyped on the GGPv3 platform, we conducted similar imputation steps to impute all animals to GGPv3. Then, we combined the imputed and physically genotyped panels (imputed $\mathrm{HD}$, imputed GGPv3, and physically genotyped HD), and finally imputed the resulting animals to sequence resolution using the sequence-based imputation reference population, described above. LINKPHASE3 [24] and Beagle 5.0 [22] were used for all phasing and imputation steps. In Beagle 5.0, we applied the default parameters except for effective population size that was set at 400 , and a window size of $20 \mathrm{Mb}$ was used except for chromosomes 7, 10,12,14, and 23, for which a $7-\mathrm{Mb}$ window size was applied because of the greater computational demands for these chromosomes, probably due to assembly and structural complexities (as previously reported [25]). Very rare variants (homozygous alternate count $\leq 5$ ) were removed by post-imputation filtering and poorly imputed variants based on the dosage $R^{2}$ statistic $\left(\mathrm{DR}^{2} ; \mathrm{DR}^{2}<0.7\right)$ were also filtered out. In total, $16,640,294$ variants remained for the GWAS and further analyses.

\section{Genotypes for the adjustment of population structure}

We used the genotyping data from the Bovine SNP50 chip platforms to account for spurious effects due to population structure. From the initial 54,708 autosomal SNPs, markers with a high missing genotype rate $(>0.01)$, a low minor allele frequency $(<0.02)$, or that deviated from the expected Hardy-Weinberg equilibrium ( $>0.15$, calculated within breed) were excluded. An additional filtering step was carried out to remove poorly imputed markers $\left(\mathrm{DR}^{2}<0.9\right)$ and markers in high linkage disequilibrium (LD) with another marker on the panel (pairwise $R^{2}>0.9$, within $1 \mathrm{Mb}$ ). After these edits, a set of 31,451 SNPs remained for subsequent analyses.

\section{Heritability estimates}

We estimated breed-specific additive and dominance heritabilities based on genomic relationship matrices (GRM) using the GCTA software [7, 26]. Additive and dominance variance components were estimated simultaneously from purebred individuals $(\mathrm{HF}=20,893$ and $J=13,184$ ), using the same set of 31,451 filtered BovineSNP50 SNPs as for population structure adjustment (see previous section). The GCTA software estimates the variance components using a restricted maximum likelihood (REML) approach. It estimates the additive heritability $\left(h^{2}\right)$ as the ratio of additive genetic variance to phenotypic variance, and dominance heritability $\left(\delta^{2}\right)$ as the ratio of dominance genetic variance to phenotypic variance. We analysed yield deviations which aggregate the herd test records that are described in the "Phenotypes" section, thus no additional records not already described were used in this analysis.

\section{GWAS}

\section{Overview of the model}

We applied a non-additive GWAS approach that is similar to that described in Reynolds et al. [12] to identify non-additive QTL for milk traits. This approach is a two-step method that leaves-one-segment-out (LOSO) and fits all other genomic SNP effects among the 31,451 SNPs to adjust for population structure, and then applies a Markov chain Monte Carlo (MCMC) method to test the effects of all imputed-to-sequence variants in the segment that had been left out, one at a time. In general, for each sequence variant the method fits the following model:

$$
\mathbf{y}=\mathbf{1} \mu+\mathbf{T b}+\mathbf{M}_{\alpha} \alpha+\mathbf{M}_{\delta} \delta+\mathbf{e},
$$

where $\mathbf{y}$ is the vector of one of the five phenotypes of interest that were pre-adjusted as described in the "Phenotypes" section; $\mu$ is the overall mean; $\mathbf{1}$ is a vector of $1 \mathrm{~s} ; \mathbf{b}$ is a vector of genotype class effects for the sequence variant of interest; $\mathbf{T}$ is the design matrix relating 
records to genotype class for the sequence variant; $\alpha$ is a vector of random additive effects of SNPs spanning the whole genome except the segment of interest such that $\boldsymbol{\alpha} \sim N\left(\mathbf{0}, \mathbf{I} \sigma_{\alpha}^{2}\right)$, and $\mathbf{I}$ is an identity matrix of order equal to the number of SNP effects and $\sigma_{\alpha}^{2}$ is the additive variance of the SNP effects; $\delta$ is a vector of random dominance effects of SNPs spanning the whole genome except the segment of interest such that $\boldsymbol{\delta} \sim N\left(\mathbf{0}, \mathbf{I} \sigma_{\delta}^{2}\right)$, and $\sigma_{\delta}^{2}$ is the dominance variance of the SNP effects; $\mathbf{M}_{\boldsymbol{\alpha}}$ and $\mathbf{M}_{\delta}$ are matrices in which each column represents the covariate values for a marker locus $([0,1,2]$ and $[0$, $1,0]$, respectively); and $\mathbf{e}$ is the vector of residual errors with $\mathbf{e} \sim N(\mathbf{0}, \mathbf{R})$, such that for a simple model based on single observations $\mathbf{R}=\mathbf{I} \sigma_{\mathrm{e}}^{2}$, and $\mathbf{I}$ is an identity matrix of order equal to the number of phenotypic records and $\sigma_{\mathrm{e}}^{2}$ is the residual error variance. Since the traits investigated here are represented by the mean of a variable number of repeated test day observations, the diagonal elements of $\mathbf{R}$ varied according to the number of observations contributing to the yield deviation. One notable contrast to the model previously implemented in Reynolds et al. [12], is that, here, we fit both additive $\left(\mathbf{M}_{\alpha}\right)$ and dominance $\left(\mathbf{M}_{\delta}\right)$ effects of the genomic markers to adjust for population structure. This modification was made to better control the inflation that was observed when analysing milk traits in a population larger than that studied in Reynolds et al. [12].

\section{Adjustment of population structure}

Five hundred samples of vectors of plausible additive and dominance SNP effects, $\widetilde{\alpha}$ and $\widetilde{\delta}$, were generated for the 31,451 SNPs using single-site Gibbs sampling based on the Bayes $\mathrm{C} 0$ algorithm implemented in the GenSel program using standard priors [27]. The fitted model omitted the Tb term from Eq. (1) and the convergence of the Markov chain of plausible SNP effects was determined using the Geweke diagnostic [28]. The LOSO approach was used to avoid fitting effects of nearby SNPs that are in linkage disequilibrium with the sequence variant being tested. The genome was partitioned into 10-Mb LOSO intervals and, for each interval, phenotypes were adjusted for the samples of SNP effects except for those within the relevant LOSO interval. This produced distinct LOSOadjusted phenotypic deviations for each $10-\mathrm{Mb}$ interval for each sample of plausible SNP effects.

\section{Association analysis}

We sampled the effects of genotype classes for each sequence variant separately, for every plausible sample of LOSO-adjusted phenotypic deviations. We obtained MCMC chains of additive and dominance genotypic effects, and standard-additive effects as contrasts of these plausible effects of genotype classes. The posterior distributions were summarised in terms of their posterior means, posterior standard deviations, and z-statistics that assumed a standard normal distribution [29]. The statistical significance of standard-additive, additive, and dominance genetic effects were evaluated using a Z-test.

\section{QTL identification, significance criteria, and annotation}

Our primary aim was to detect non-additive QTL, thus we declared variants as significant if the dominance genotypic effect, $d$, passed a false discovery rate (FDR) threshold of $1 \times 10^{-3}$. For each phenotype, this FDR threshold was calculated using q-values [30] as implemented in the qvalue package in R [31]. Since we were particularly interested in medium- to large-effect QTL, only the loci with effect sizes ( $a$ or $d$ ) greater than 5\% of the phenotypic standard deviation of the trait were considered for further downstream analyses. We calculated the dominance coefficient $k=\frac{d}{|a|}$ for each significant QTL to characterise the underlying non-additive mechanism where $k \approx 0$ represents a completely additive locus, $k \approx 1$ a completely recessive locus, $k<1$ a partially dominant locus, and $k>1$ an over-dominant locus. For standard additive effects, $\alpha$, we used GCTA-COJO [32] to detect tag variants for QTL identified in our standard additive GWAS. The GCTA-COJO routine uses LD structure and GWAS summary statistics to iteratively identify significant QTL at the FDR threshold of $1 \times 10^{-3}$.

We used sequence annotations from variant effect predictor (VEP; Ensembl 97, [33]) to highlight mutations that might be responsible for the non-additive QTL identified, and then used SIFT scores to evaluate the potential impact of any missense mutations on protein function [34]. To assess the quality of VEP-derived variant annotations and ensure that the predicted missense and nonsense variants intersected expressed exons, we manually visualised mammary RNA-seq alignments as described in Reynolds et al. [12] using the Integrative Genomics Viewer (IGV) [35]. These analyses confirmed that, for the three non-sense candidate mutations identified in ITGAL, LRCH4, and RBM34, all appeared to encode valid premature stop variants, and in the case of the LRCH4 mutation, its position that is adjacent to the exon 3 splice acceptor boundary suggested that the variant might also have splicing consequences. We also manually inspected genome sequence alignments representing the non-additive QTL regions in animals of contrasting QTL genotyping (i.e. those carrying opposing alleles of the QTL tag SNPs), to look for possible gene-disrupting structural variants in these regions.

\section{Iterative GWAS}

We were interested in determining if multiple dominance QTL might segregate at associated loci, thus we 
implemented an iterative GWAS approach to differentiate QTL. First, we identified on each chromosome the variants with an FDR lower than the threshold. Next, we adjusted the phenotype for the effects of the genotype classes of the most significant variant (or candidate causal variant if identified) and then re-ran the GWAS model on the chromosome of interest using the adjusted phenotype. This process was iteratively repeated until no significant QTL remained on the chromosome.

\section{Results}

\section{Heritabilities of lactation traits}

First, we estimated the additive and dominance heritabilities for each phenotype within each breed to investigate the additive and non-additive genetic architecture of each trait. These results (Table 1) show that the dominance heritabilities were far outweighed by the additive heritabilities. This was not surprising as the values presented are of similar magnitude to those reported for other traits and populations in the literature $[9,36]$. Milk fat yield in Jersey cows had the highest dominance heritability at 0.074 , and milk protein percentage in HolsteinFriesian cows had the lowest dominance heritability at 0 . It should be noted that there was a clear contrast between the relative heritabilities of milk composition and milk yield traits, with milk composition traits displaying high additive heritabilities but near to zero dominance heritabilities, whereas milk yield traits displayed lower additive heritabilities but higher dominance heritabilities (Table 1).

\section{GWAS for lactation traits}

We performed GWAS across the five milk traits of interest, namely milk volume, milk protein yield, milk fat yield, milk protein percentage, and milk fat percentage, to identify non-additive QTL (Fig. 1). Both additive and dominance effects are included in these plots, and the iterative analysis identified 23 dominance QTL signals that were above the FDR threshold of $1 \times 10^{-3}$. Some of the QTL were identified for multiple traits. These dominance QTL included 10, 11, 12, 8, and 7 QTL represented by $4618,2706,8525,8987$, and 5800 significant variants for milk volume, milk protein yield, milk fat yield, milk protein percentage, and milk fat percentage, respectively. The QTL spanned 13 discrete autosomes. After the iterative COJO analysis, standard additive GWAS identified 217, 152, 142, 673, and 457 QTL for milk volume, milk protein yield, milk fat yield, milk protein percentage, and milk fat percentage, respectively.

\section{Dominance QTL}

We identified 15 significant dominance QTL for milk yield traits, and 11 for milk composition traits (Table 2) and (see Additional file 1: Table S1). Twelve of the 15 milk yield dominance QTL had recessive effects and were located on chromosomes 2, 4, 5, 8, 12, 25, 28, or 29 . Seven of these signals did not appear to have been previously reported, whereas the remainder were highlighted in our analysis [12] of growth and developmental traits in a population that overlapped with that described here. Eight of the 11 milk composition dominance QTL presented partial dominance effects, of which six were identified in our previously published additive GWAS (see Additional file 1: Table S1). Figure 2a compares the minor allele frequency and the size of the effect of the dominance components for all these loci. Interestingly, milk composition QTL appeared to be tagged by high minor allele frequency variants with comparatively small effects, whereas milk yield QTL were tagged by variants that had low minor allele frequencies and large effects. The type of effects also appeared to differ between traits (Fig. 2b), where milk yield traits were mostly impacted by recessive QTL, whereas milk composition traits near-exclusively presented QTL showing partial dominance.

\section{Identification of candidate causal mutations}

Given that the recessive milk yield QTL potentially represented novel bovine disorders, we prioritised these QTL for further investigation and selected those for which the dominance coefficient $(k)$ was near $1(0.7<k<1.3)$. We used sequence annotations from VEP to highlight the mutations that might be responsible for these effects (Ensembl 97, [33]), i.e. pinpointing variants that were in strong to moderate $\mathrm{LD}\left(\mathrm{R}^{2}>0.7\right)$ with the lead variant

Table 1 Heritability estimates for lactation traits

\begin{tabular}{lllll}
\hline Trait & $\boldsymbol{h}_{\mathrm{HF}}^{\mathbf{2}}(\mathrm{SE})$ & $\boldsymbol{\delta}_{\mathrm{HF}}^{\mathbf{2}}(\mathrm{SE})$ & $\boldsymbol{h}_{\mathbf{J}}^{\mathbf{2}}(\mathrm{SE})$ & $\boldsymbol{\delta}_{\mathbf{J}}^{\mathbf{2}}(\mathrm{SE})$ \\
\hline Milk volume & $0.296(0.010)$ & $0.044(0.007)$ & $0.312(0.012)$ & $0.064(0.009)$ \\
Milk fat yield & $0.261(0.010)$ & $0.059(0.008)$ & $0.232(0.012)$ & $0.074(0.010)$ \\
Milk protein yield & $0.235(0.009)$ & $0.053(0.008)$ & $0.236(0.012)$ & $0.073(0.010)$ \\
Milk fat percentage & $0.700(0.007)$ & $0.006(0.004)$ & $0.616(0.010)$ & $0.015(0.006)$ \\
Milk protein percentage & $0.642(0.008)$ & $0(0.005)$ & $0.636(0.010)$ & $0.005(0.005)$ \\
\hline
\end{tabular}

$h^{2}$ : additive heritability: $\delta^{2}$ : dominance heritability; HF: Holstein-Friesian, J: Jersey; SE: standard error 


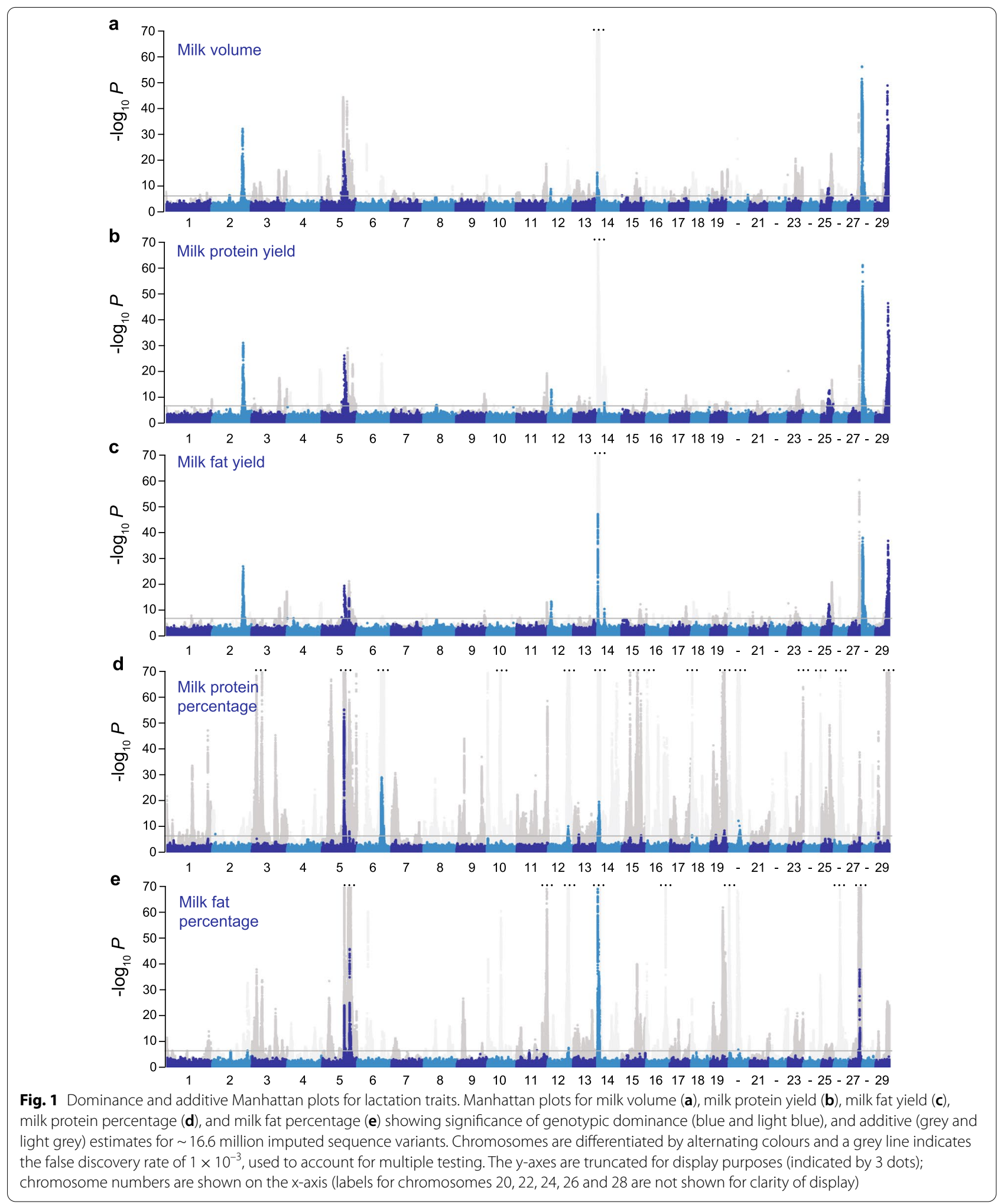




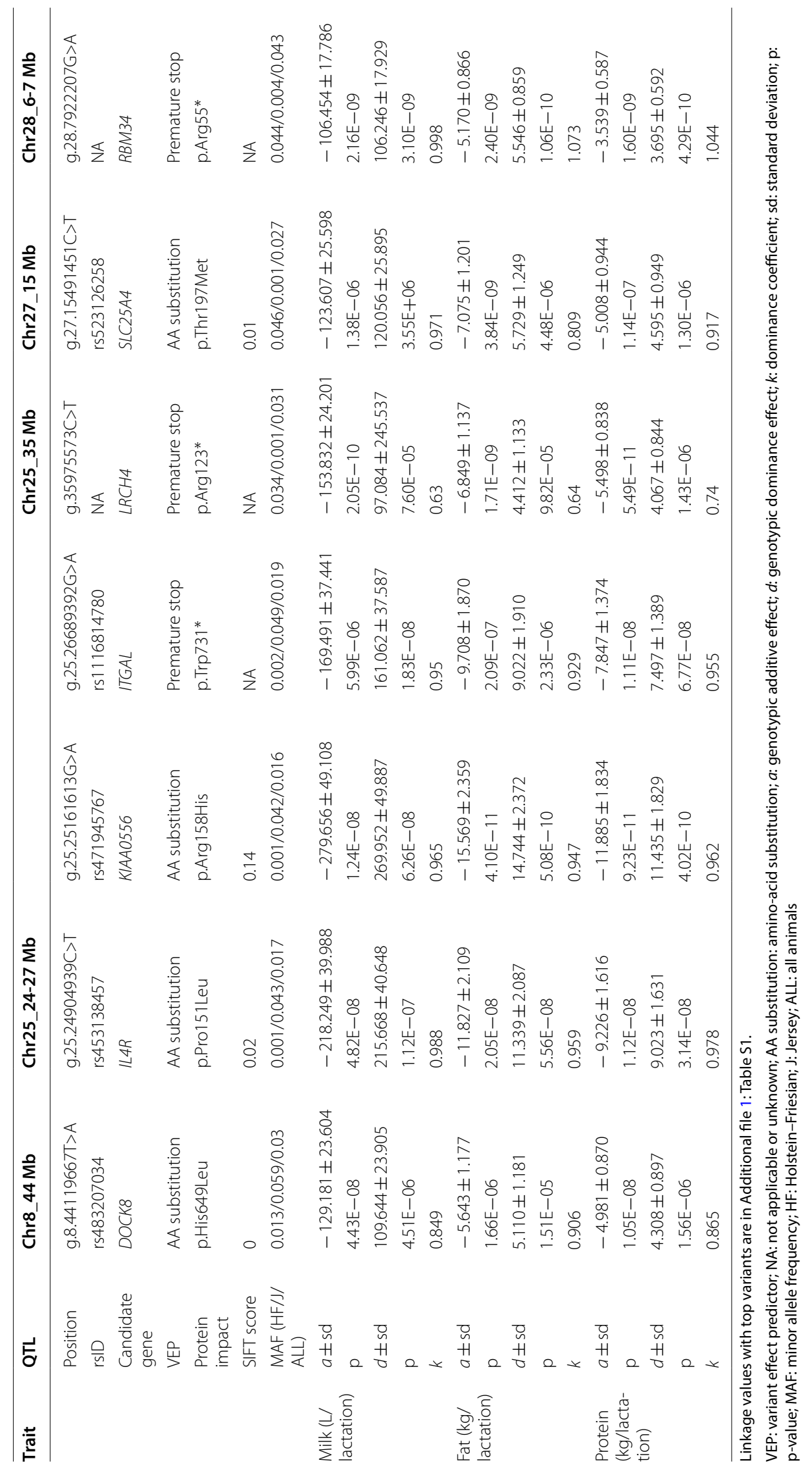


a)

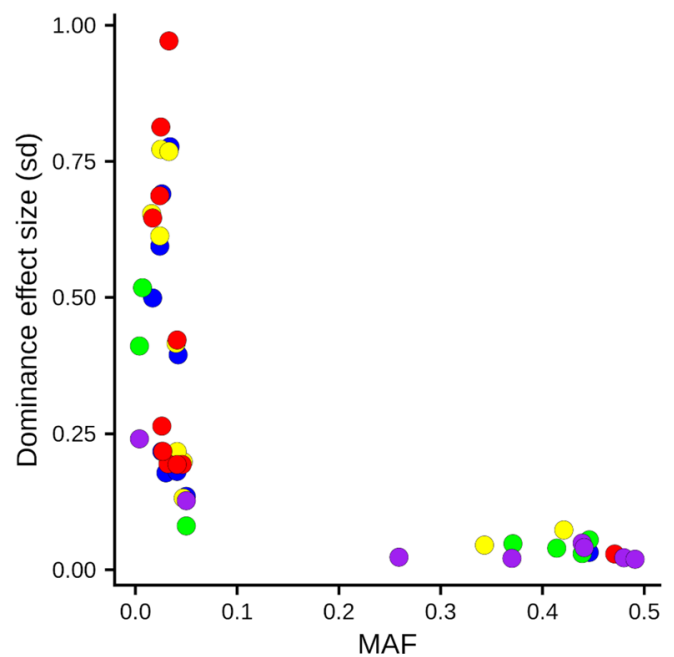

b)

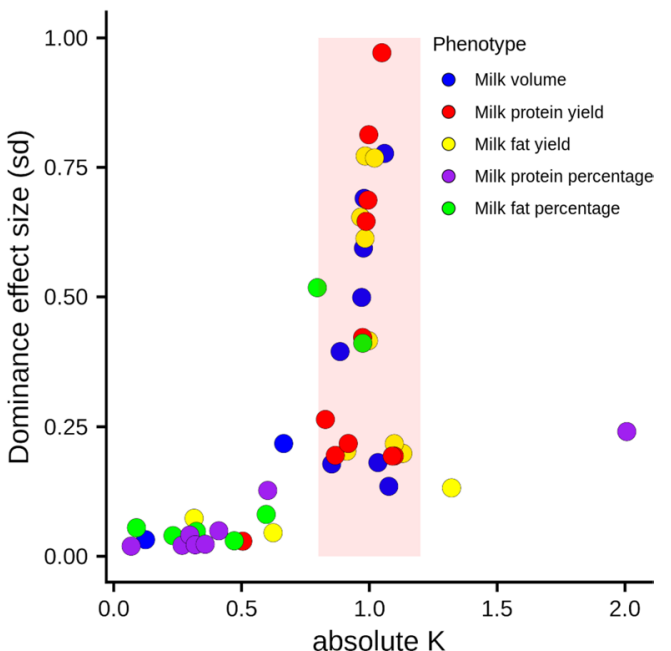

Fig. 2 Plots presenting the genetic architecture of significant dominance QTL from GWAS on milk volume, milk protein yield, milk fat yield, milk protein percentage, and milk fat percentage. The plots contrast the minor allele frequency (MAF) against the dominance effect size (a), and the absolute value of $k$, where $k=d /|a|$, against the dominance effect size $(\mathbf{b})$

per locus, and that were also predicted to alter or disrupt protein function. Furthermore, we manually investigated each QTL by visualising whole-genome sequence alignments that corresponded to animals with contrasting QTL genotypes. This step was performed to identify obvious structural mutations that were not detected by automated variant calling, i.e. those intersecting genes that could be similarly expected to modify or ablate gene function. However, we did not identify any structural variants that tagged QTL. It should be noted that these methods focussed only on protein-coding variants as candidates since, for recessive signals at least, we consider that protein altering mutations are primary candidates given the loss of function mechanism assumed to underlie recessive QTL. However, this does not preclude the involvement of regulatory variants, which we did not consider in our study. We identified five novel recessive QTL (including one near-significant recessive QTL), and several other previously identified recessive effects attributed to mutations in the PLCD4, FGD4, MTRF1, GALNT2, DPF2, and MUS81 genes [12]. Figure 3 presents the position, regional LD, and association statistics for the QTL that are novel to this paper. Additional file 1: Table S1 shows all significant QTL identified, including those that are not described in detail here.

\section{Chromosome 8}

Chromosome 8 presented a significant signal at $45 \mathrm{Mb}$ for milk protein yield and milk fat yield. The most significant variants for these signals (g.45878531A $>C$ and g.45880948C $>T)$ were in strong $L D\left(R^{2}=0.99\right)$, and an annotated missense variant (g.44119667T>A, rs483207034) was in high LD with both of the top-associated variants $\left(R^{2}=0.85\right.$ and 0.85 , respectively; Fig. $\left.3 a\right)$. This variant in the $D O C K 8$ gene results in an amino acid (p.His649Leu) change and has a predicted deleterious impact $(\mathrm{SIFT}=0)$.

\section{Chromosome 25}

A dispersed QTL signal was found on chromosome 25, spanning 24-27 Mb for the three lactation yield traits. The region presented different top-associated variants for milk fat yield (g.25921991AT>T) and milk protein yield and volume traits (g.27868969C $>$ T). Variant effect prediction highlighted three candidate causal mutations in the region. These included a p.Pro151Leu substitution in the IL4R gene (g.24904939C $>\mathrm{T}$, rs453138457) with $\mathrm{R}^{2}=0.74$, and 0.62 , for the milk fat and milk protein/milk volume top variants, respectively, another missense variant (p.Arg158His) in the KIAA0556 gene (g.25161613G>A, rs471945767) with $\mathrm{R}^{2}=0.89$, and 0.74 , respectively, and a nonsense variant (p.Trp731*) in the ITGAL gene (g.26689392G>A, rs1116814780) with $\mathrm{R}^{2}=0.76$, and 0.70 , respectively (Fig. $3 \mathrm{~b}$ ). Although all these variants represented plausible candidates to explain the QTL, we were not able to distinguish between the candidates through iterative analysis, since when any one of these candidates was fitted, the majority of the association for any of the other candidates was removed at this locus.

A second signal for protein yield on chromosome 25 was observed at $35 \mathrm{Mb}$. That locus maintained its 
a)

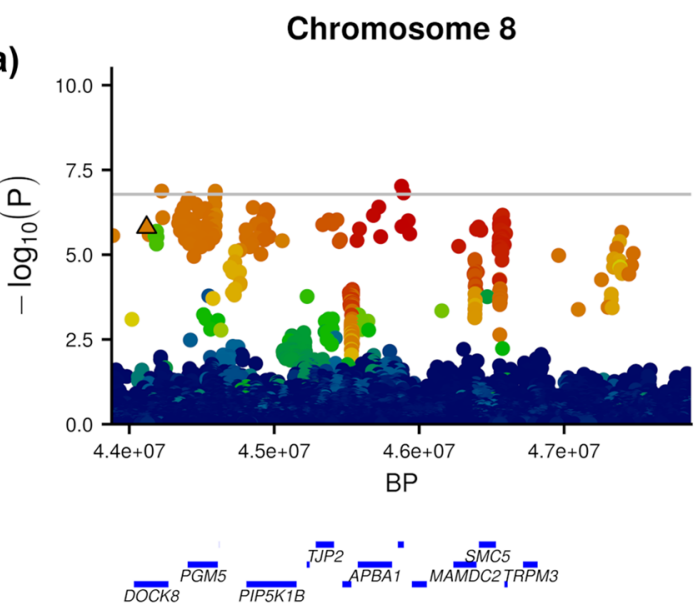

c)

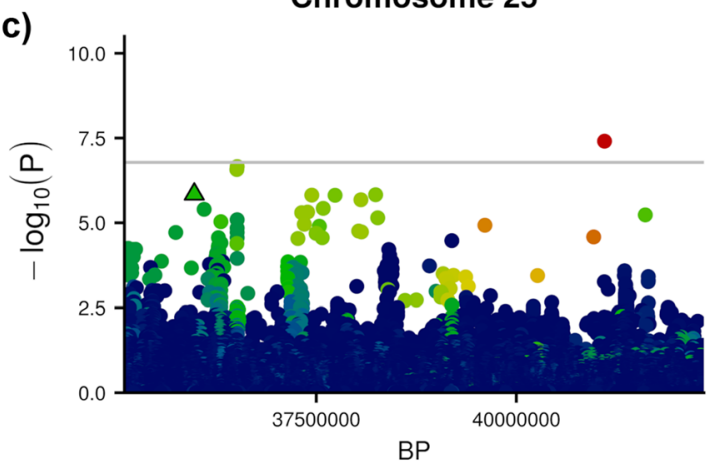

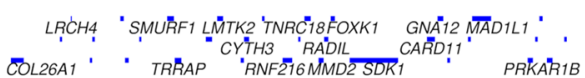

b)

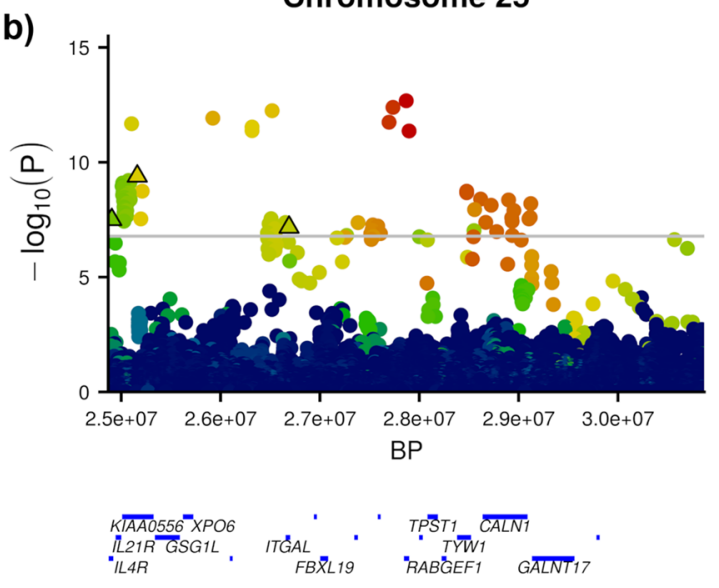

d)

Chromosome 27

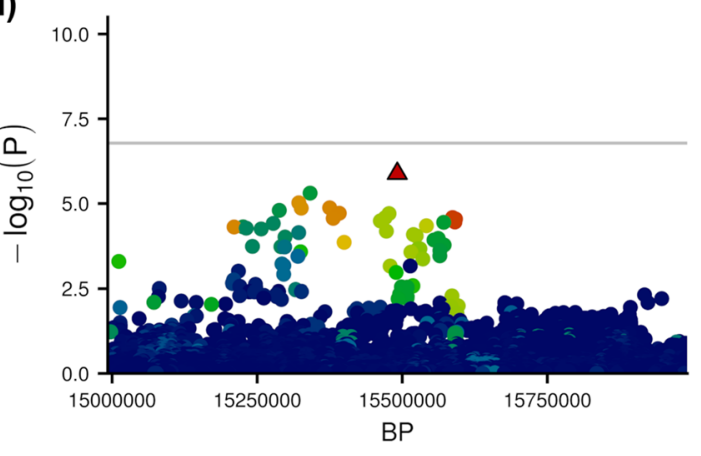

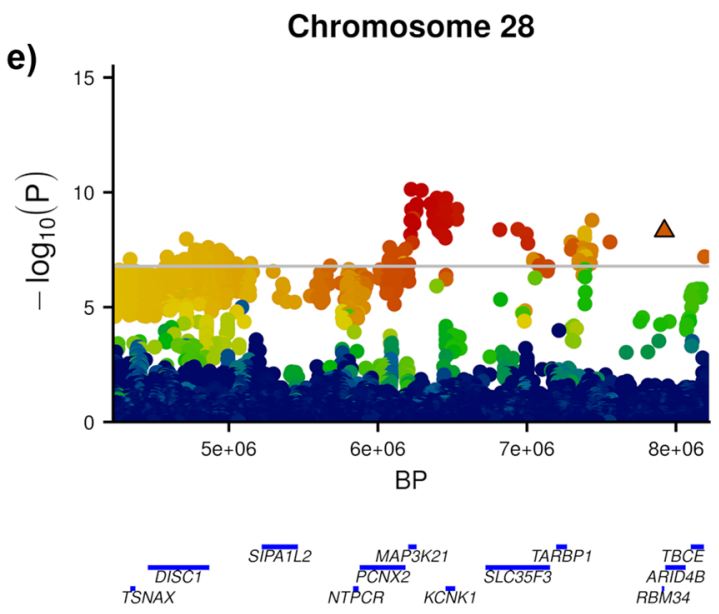

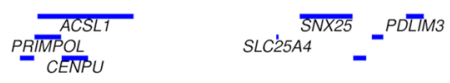

Fig. 3 Manhattan plots for the five novel milk protein yield QTL representing the chr8:44Mbp (a), chr25:24-27Mbp (b), chr25:35Mbp (c), chr27:15Mbp (d), and chr28:7Mbp (e) loci. Variants are coloured by $L D\left(R^{2}\right)$ values with the top tag variant per locus, protein coding variants are shown as outlined triangles. Gene tracks are presented below each plot based on Ensembl 97, where gene names have been filtered on size 
significance after accounting for the QTL on chromosome 25 at $24-27 \mathrm{Mb}$ through iterative analysis, suggesting that it was a different effect. The locus presented a strong candidate causative mutation that could underlie the effect, i.e. a stop gain mutation (g.35975573C $>$ T; Arg123*) in the LRCH4 gene that was the third most highly associated variant at this locus overall (Fig. 3c). We observed a mostly recessive effect for this variant $(k=0.74)$, with the animals that carried the heterozygous and homozygous alternate genotypes producing $1.44 \mathrm{~kg}$, and $11.21 \mathrm{~kg}$ less milk protein per lactation compared to the homozygous reference genotype. When g.35975573C $>\mathrm{T}$ was fitted as a fixed effect, the significance of the QTL was removed, and no other QTL was detected on the chromosome (see Additional file 2: Fig. S1).

\section{Chromosome 27}

We observed a signal at $15 \mathrm{Mb}$ on chromosome 27 for milk protein yield. Although this did not exceed our q-value FDR threshold of $1 \times 10^{-3}$ (equivalent to $\mathrm{p}$-value $=1.65 \times 10^{-7}$ ), this signal was notable given that the top variant (g.15491451C $>$ T; rs523126258, $\mathrm{p}$-value $\left.=1.30 \times 10^{-6}\right)$ is a predicted deleterious missense mutation (p.Thr197Met) in the SLC25A4 gene. Figure 3d shows a Manhattan plot for this region.

\section{Chromosome 28}

We previously reported a major recessive bodyweight QTL on chromosome 28 that corresponds to a likely causative splice acceptor mutation in the GALNT2 gene (g.2281801G >A) [12]. This QTL was observed in the current analysis and impacted all three milk yield traits. However, iterative association analysis revealed a secondary QTL that is located approximately $4 \mathrm{Mb}$ downstream of the GALNT2 mutation at Chr28:6-7 Mb (top variant at g.6223350G>A). This residual signal highlighted a stop-gain non-sense mutation ( $\mathrm{g} .7922207 \mathrm{G}>\mathrm{A})$ that is strongly linked to the g.6223350G $>A$ variant $\left(R^{2}=0.89\right.$; Fig. 3e). This stop-gain mutation (p.Arg55*) is located in the $R B M 34$ gene, appears to be in linkage equilibrium with the GALNT2 causal mutation $\left(\mathrm{R}^{2}<0.001\right)$, and was not associated with bodyweight in our previous analysis $(\mathrm{p}=0.37[12])$. A second GWAS iteration on chromosome 28 (fitting both GALNT2 and RBM34 mutations as fixed effects) did not reveal any other significant QTL on the chromosome (see Additional file 3: Fig. S2).

\section{Comparison between lactation and growth trait recessive QTL}

We were interested in determining whether the novel recessive candidate causal mutations identified here had effects on the growth and developmental traits investigated in our previous study [12]. Here, we assessed the association statistics of these variants reported in that study, and while none of the novel mutations reached statistical significance (and would have thus been reported as part of that analysis), some did display apparent recessive mechanisms of moderate effect size. This suggests that, with increased sample sizes, these variants may present significant effects on growth traits. Notably, the mutation in KIAA0556 was one of the most strongly associated variants for body condition score in that study, presenting the 10th smallest dominance $\mathrm{p}$-value of the $\sim 16$ million variants tested in that analysis. Additional file 1: Table S2 includes the association statistics for five of the seven candidate causal mutations presented above (the ITGAL and SLC25A4 mutations were not captured in the genotype dataset reported by Reynolds et al. [12]). All of the novel candidate mutations highlighted in Reynolds et al. [12] were also associated with lactation traits (see Additional file 1: Table S1) except for the MYH1-disrupting structural variant which was only associated with body condition score in that study.

\section{Dominance QTL for composition traits}

In addition to the recessive QTL identified for milk yield traits, we also identified dominance QTL for milk composition traits. We investigated these effects and observed several partial dominance QTL that are in close proximity to previously described additive loci. The tag variants of these QTL were adjacent to the following genes: CSF2RB [37], MGST1 [17], DGAT1 [13], GHR [14], GPAT4 [16], and PICALM [38] and, in each case, these variants were in high $L D\left(R^{2}>0.8\right)$ with previously identified causal and/or tag variants (see Additional file 1: Table S1).

Milk protein percentage presented multiple dominance QTL on chromosome 6 within the 80 to $85 \mathrm{Mb}$ region (see Additional file 1: Table S1). Among these QTL, the most significant variant (g.84112451C $>$ A) showed a partial dominance effect. Unlike in the above examples, we did not identify any very strongly linked candidate mutation although this variant was in moderate LD with a previously proposed causative variant in the CSN1S1 
gene $\left(R^{2}=0.53\right.$; p.Glu192Gly mutation; g.85427427A>G) [39]. Chromosome 12 presented a significant dominance QTL, for which we observed a partial dominance effect at $68 \mathrm{Mb}$ for milk protein percentage with the top variant at g.68763031T> TG. As observed for the chromosome 6 locus, no particularly obvious candidate causal variant or gene was identified that might account for that signal.

\section{Comparison between the additive and dominance GWAS results}

Figure 4 compares the minor allele frequency (MAF) and the effect sizes between homozygous genotypes across all traits and genetic mechanisms. As expected, we observed many more additive QTL than dominance QTL across all traits. On the one hand, it is noteworthy that the mutations detected via dominance GWAS for milk yield traits had very large effects compared to the additive QTL detected for these traits, and most of them had a recessive effect. On the other hand, the largest effects observed for the two milk composition traits were mostly additive QTL, and dominance effects tended to have high MAF and presented mostly partial dominance effects.

\section{Discussion}

Our results highlight the presence of many non-additive QTL for milk traits in cattle. The majority of these signals for milk yield traits present recessive QTL, that involve five novel loci and several previously described recessive loci [12]. Although the milk protein percentage and milk fat percentage traits also yielded many dominance GWAS signals, most of them correspond to partially dominant effects that are attributable to previously reported additive QTL.

\section{Different trait classes present contrasting additive} and non-additive genetic architectures

One remarkable observation from our study is the apparent difference in additive and non-additive genetic architectures between milk yield traits and milk composition traits. Dominance heritabilities for the milk yield traits ranged from 3 to $7 \%$, whereas for the milk composition traits they were zero or near zero. In contrast, the additive heritabilities ranged from 23 to $31 \%$ for the milk yield traits and from 64 to $70 \%$ for the milk composition traits. These findings are consistent with those of Sun et al. [9] who report similar additive and dominance heritabilities,

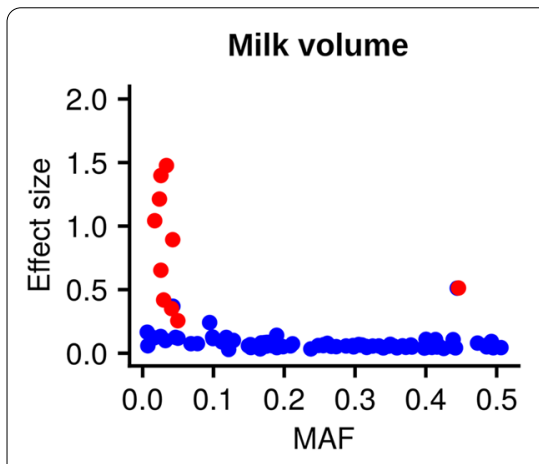

Milk fat percentage

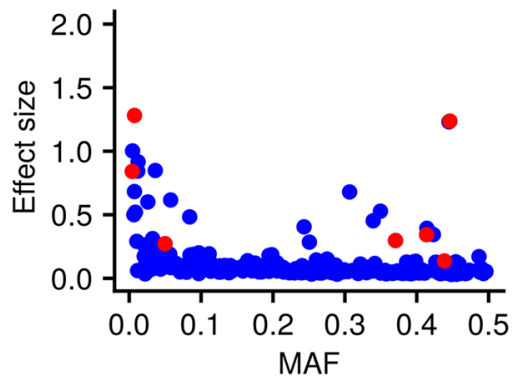

Milk fat yield

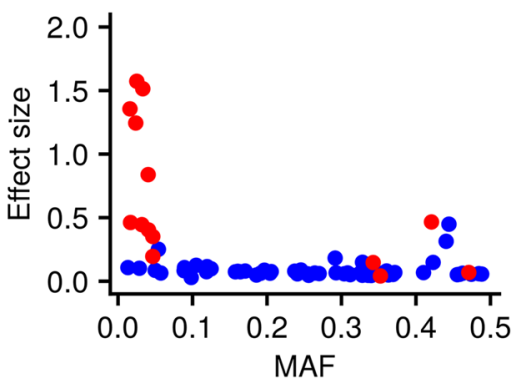

Milk protein percentage

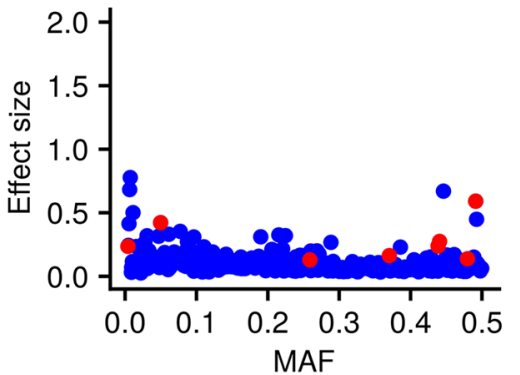

Fig. 4 Plots contrasting minor allele frequency (MAF) and the absolute effect size between homozygote genotype classes (effect size) for additive (blue) and dominance (red) QTL detected via GWAS across lactation traits 
and suggest that dominance, in particular recessive mechanisms, may play a bigger role in the regulation of milk yield traits than that of composition traits.

These differences in the genetic architecture of the milk traits investigated in this study were also observed when the properties of individual dominance QTL were compared between milk yield and milk composition traits. The majority of the dominance QTL identified for milk yield traits had recessive genetic effects, while the majority of the milk composition traits had partial dominance effects. Furthermore, the dominance QTL for milk yield traits were characterised by low MAF and large effect sizes, whereas those for milk composition traits were characterised by high MAF and comparatively smaller effect sizes. We hypothesize that these observations reflect the way in which different traits may represent underlying recessive syndromes-i.e., their utility as proxies for genetic disorders. Among all the recessive QTL detected in our study, a subset of these had previously been validated as representing new genetic disorders [12]. Although we did not investigate the novel recessive loci in this study with the same rigour as those analysed in Reynolds et al. [12], their very large, uniformly negative effects suggest that at least some of them will be similarly validated. Notably, none of these loci (new or old) show substantial effects on milk composition, suggesting that milk fat and protein percentage traits do not readily reflect recessive effects. This finding can be rationalised by the comparatively broad range of biological processes expected to impact milk yield traits (or the growth and development traits investigated in Reynolds et al. [12]), where the energy demands of lactation (or growth) may manifest a wide range of other organismal stresses. In contrast, the relative composition of milk components likely represents a narrower spectrum of mammary-specific biological mechanisms, and thus we hypothesise that these traits are less able to serve as proxies of animal fitness.

It should be acknowledged that given that protein yield and fat yield are the products of milk volume and their respective percentages, these traits are not independent. We observed that the variance components and the genetic architectures of milk fat yield and milk protein yield are more comparable to milk volume than their respective composition traits.

\section{Previous studies highlighting recessive effects on quantitative traits}

As discussed above, we recently reported an investigation of growth and developmental traits that identified non-additive QTL using similar approaches to those presented here [12]. That study demonstrated how quantitative traits can be used as proxies to map genetic disorders without prior disease identification. In doing so, we highlighted several recessive QTL represented by variants in the PLCD4, FGD4, MTRF1, GALNT2, DPF2, and MUS81 genes, each with large effects on bodyweight and other quantitative traits. The work presented in the current paper builds on those findings; we identified many of the same recessive mutations as well as several additional recessive QTL. Some of these additional QTL displayed moderate but not significant recessive effects for growth traits and their discovery may be assumed to reflect the increased sample sizes leveraged in the current study. These findings suggest that milk yield traits might also be used to represent whole-animal health, and since lactation measurements are more routinely derived than bodyweight phenotypes (at least in bovine dairy systems), these likely represent a more accessible phenotype relevant to a larger number of international evaluation systems.

Few studies other than Reynolds et al. [12] have highlighted major recessive effects using quantitative trait data. Although non-additive GWAS with large sample sizes have been performed in cattle $[11,36]$, the low density of the SNP arrays used in those earlier studies may have hampered the ability to directly resolve candidate causative variants [12]. This challenge arises due to the different LD properties between causal and observed variants for additive and non-additive QTL, such that the variance that an observed variant can explain decreases by $\mathrm{R}^{2}$ for additive $\mathrm{QTL}$, and by $\mathrm{R}^{4}$ for dominant or recessive QTL. This means that the observed tag variants need to be more closely linked to the causal dominance variants to capture the QTL $[40,41]$. The fact that major deleterious alleles are also likely to be infrequent compounds this problem. Under Hardy-Weinberg expectations where $\mathrm{p}^{2}+2 \mathrm{pq}+\mathrm{q}^{2}=1$, the number of rare allele homozygotes $\left(\mathrm{q}^{2}\right)$ decreases exponentially as allele frequency decreases. Practically, this means very large sample sizes are needed to represent rare allele homozygotes, where at 1\% MAF, 10,000 individuals would be expected to present a single homozygote (with 1,000,000 individuals required at $\mathrm{MAF}=0.1 \%$ ). However, as sample sizes and high-density genotyping platforms begin to permit such analyses, we anticipate similar such studies in other populations to begin to appear. One recent, noteworthy such study has suggested the importance of recessive variants in the context of male fertility and semen traits in cattle [42]. In that study, recessive QTL and candidate causal mutations were identified in several genes including a missense variant in the SPATA16 gene. That discovery was based on imputed genotypes at high density (the Illumina BovineHD platform), but the size of the studied population was quite small $(\mathrm{N}=3736$ bulls). It is likely that the discovery of these QTL was partly aided by the 
remarkable frequency of the deleterious haplotypes identified in that study, presenting allele frequencies ranging from 9 to $34 \%$ [42].

\section{Recessive QTL of interest}

Although many non-additive signals were identified in our study, we were particularly interested in the recessive QTL with large effects, given that these might represent underlying genetic disorders. We highlighted protein-coding variants as candidates because we considered these to be the most probable causal variants, but we acknowledge this is a relatively simple approach and that regulatory or unidentified structural variants may alternatively underlie these recessive QTL. These caveats aside, the five novel recessive QTL on chromosomes 8, 25,27 , and 28 are presented and discussed below.

\section{Chromosome 8-DOCK8}

Our results present a missense mutation in the DOCK8 gene as potentially having a deleterious recessive impact on milk yield traits. The QTL appears to operate in a completely recessive manner, with the DOCK 8 variant present at low allele frequencies in each breed (Holstein-Friesian $\mathrm{MAF}=0.013$ and Jersey $\mathrm{MAF}=0.059$ ). The DOCK8 gene encodes dedicator of cytokinesis 8 , a member of the DOCK180 family of guanine nucleotide exchange factors, which influences intracellular signalling networks and is important in immune responses and lymphocyte regulation in humans and mice [43]. Recessive mutations in DOCK8 have been associated with the hyper immunoglobulin E syndrome which leads to the onset of an immunodeficiency disease combined with other health complications [44]. In mice, compromised immune responses are also observed including negative impacts on B cell migration [45], and $\mathrm{T}$ cell migration and viability $[46,47]$. DOCK8 variants have not previously been associated with cattle performance traits, but if this missense mutation underlies the QTL on chromosome 8 , we hypothesized that it could act through similar negative impacts on the immune system. Under this hypothesis, it is unknown whether the effects on lactation are due to mammary immune function or secondary impacts. However, given that higher levels of circulating immunoglobulins $\mathrm{E}$ and lymphocyte profiling can indicate DOCK8 deficiency in humans $[44,48]$, it would be interesting to sample and profile homozygous animals to definitively establish the causality of the DOCK8 missense mutation for this QTL.

\section{Chromosome 25-IL4R, KIAA0556, ITGAL}

The QTL identified on chromosome 25 at $24-27 \mathrm{Mb}$ presented three candidate mutations in the IL4R, KIAA0556, and ITGAL genes. The $I L 4 R$ gene encodes the interleukin
4 receptor, which is a transmembrane protein involved in immune responses in humans [49]. The KIAA0556 gene is associated with microtubule regulation in humans, and KIAA0556 knockout mutations in humans and mice have been associated with Joubert syndrome, a neurological disorder [50]. The ITGAL gene encodes the integrin alpha $\mathrm{L}$ chain, and loss of function variants in this gene have been associated with compromised immunity including increased susceptibility to infection to Salmonella in mice [51]. Given that the iterative association analysis failed to prioritise one of these variants over the other, it is unknown which of these variants might be responsible for the QTL, and our focus on protein-coding variants as candidates may have also overlooked alternative noncoding or structural mutations. These variants are nevertheless in moderately strong, though not in perfect LD (maximum pairwise $\mathrm{R}^{2}=0.79$ ), thus physical genotyping for fine mapping and future functional testing should help to resolve the identity of the gene (or genes) underpinning this QTL.

\section{Chromosome 25-LRCH4}

Although iterative GWAS did not resolve candidates in the above example, this approach did highlight a second QTL on chromosome 25 represented by a nonsense mutation in the $\mathrm{LRCH} 4$ gene, which encodes leucine-rich repeats and calponin homology containing protein 4 . It regulates the signalling of toll-like receptors (TLR) and has been shown to influence innate immune responses in mice [52]. In that study, researchers showed that $\mathrm{LRCH4}$ silenced cells presented a reduced expression across proinflammatory cytokines produced in the TLR4 pathway, most notably in that of IL-10 and MCP-1. We hypothesise that the $\mathrm{LRCH} 4$ knockout mutation identified in our study may have negative impacts on the innate immunity of cattle, and that those impacts could lead to the recessive effects we observed on milk volume, milk fat yield, and milk protein yield.

\section{Chromosome 27-SLC25A4}

While non-significant at the genome-wide level (cf. $\mathrm{p}=1.65 \times 10^{-7}$ vs $\left.\mathrm{p}=1.30 \times 10^{-6}\right)$, the locus on chromosome 27 at $15.5 \mathrm{Mb}$ presented a conserved amino acid mutation in the SLC25A4 gene as the lead associated variant and was therefore of interest. This variant demonstrated a complete recessive effect on all three lactation yield traits. The SLC25A4 (solute carrier family 25 member 4) gene encodes the adenine nucleotide translocator (Ant1) protein, responsible for the translocation of ATP and ADP between the cytoplasm and mitochondria. In mice, SLC25A4 knockouts result in mitochondrial myopathy and cardiomyopathy, and severe intolerance to exercise [53]. Similarly, in humans, childhood-onset 
mitochondrial disease and exercise intolerance have been observed for both dominant [54] and recessive mutations [55] in SLC25A4. Given the implication that mitochondrial functional deficits might underlie the negative lactation effects highlighted in the current study, it would be intriguing to examine the phenotypes of homozygous cows further in this context.

\section{Chromosome 28-RBM34}

At first glance, the strong associations with the lactation yield traits on chromosome 28 might reasonably be attributed to the previously reported splice site mutation in GALNT2 [12]. However, when this mutation was fitted as a covariate in our iterative GWAS, a secondary signal was observed, highlighting a nonsense mutation in the $R B M 34$ gene as potentially responsible for the effect. The $R B M 34$ gene encodes an RNA recognition motif protein with an RNA-binding domain. The literature on RBM34 in humans or model organisms is scarce, with limited implication of the gene in embryonic stem cell differentiation [56]. Here, we observed a predicted homozygous knockout of RBM34 that may influence milk volume, milk protein yield, and milk fat yield in a recessive manner, although its status as a largely uncharacterised RNAbinding protein leaves little room for speculation as to how these effects might manifest. Mechanism aside, the identification of two co-locating, yet uncorrelated recessive QTL demonstrates the utility of using iterative GWAS approaches, given that conventional analysis would likely fail to differentiate these effects. We note that other researchers have observed effects on lactation at the 6-10 Mb locus [57]. However, the $\mathrm{LD}\left(\mathrm{R}^{2}\right.$ with $R B M 34=0.04, G A L N T 2=0.02$ ) between the tag variant identified by Raven et al. [57] (rs41607517) and the nonsense mutations identified here is very low, which suggests that they are different effects.

\section{Previously described additive QTL present partial dominance}

We observed several partial dominance QTL that are closely linked to previously described QTL identified from standard additive analyses. As presented in Additional file 1: Table S1 we identified dominance components in high LD with variants associated with the CSF2RB [37], MGST1 [17], DGAT1 [13], GHR [14], AGPAT6 [16], PLAG1 [58, 59], and PICALM [38] genes (and in moderate LD with a variant in the CSN1S1 gene [39]). These partial dominance associations were mostly identified in milk composition traits. These observations suggest that many well-known major-effect QTL that are identified in additive GWAS' incorporate some level of non-additivity, in agreement with the analyses of milk traits reported by Jiang et al. [11,36].

\section{Conclusions}

In this study, we have highlighted that different classes of lactation traits (yield compared to composition traits) present different additive and non-additive genetic architectures. We speculate, that these differences derive from dissimilarities in the cellular and molecular manifestation of these traits, and although milk yield traits have comparatively low additive heritabilities, these traits may better reflect whole-animal energy and fitness status and be a better proxy of a wider range of underlying biological disorders. At the single locus level, we identified five QTL presenting seven candidate causative variants in the $D O C K 8, I L 4 R$, KIAA0556, ITGAL, LRCH4, SLC25A4, and RBM34 genes, highlighting medium- to large-effect recessive variants that may provide future opportunity for diagnostic testing and animal improvement.

\section{Supplementary Information}

The online version contains supplementary material available at https://doi. org/10.1186/s12711-021-00694-3.

Additional file 1: Table S1. Association statistics for most significant variants. Table including association statistics for the most highly associated variant for each QTL identified through GWAS. Table S2. Association statistics for growth and developmental traits. Table including association statistics for five candidate causal mutations in the DOCK8, IL4R, KIAA0556, $L R C H 4$, and RBM34 genes for growth and developmental traits [12].

Additional file 2: Figure S1. Iterative Manhattan plots for milk-protein yield on chromosome 25. Blue indicates the candidate causal variants in genes; IL4R, KIAA0556, and ITGAL, and red indicates the candidate causal variant in the $\mathrm{LRCH} 4$ gene. A grey line indicates the false discovery rate of $1 \times 10^{-3}$, used to account for multiple testing.

Additional file 3: Figure S2. Iterative Manhattan plots for milk-protein yield on chromosome 28. Blue indicates the candidate causal variant in the GALNT2 gene, and red indicates the candidate causal variant in the RBM34 gene. A grey line indicates the false discovery rate of $1 \times 10^{-3}$, used to account for multiple testing.

Acknowledgements

We are grateful for the use of New Zealand eScience Infrastructure (NeSI) highperformance computing facilities as part of this research. We are additionally grateful to the funders of this research.

\section{Authors' contributions}

EGMR, DJG, and MDL conceived and designed the experiments. EGMR, TL, KT, $\mathrm{YW}, \mathrm{CSH}, \mathrm{BLH}, \mathrm{DJG}$, and MDL performed or assisted statistical analysis. EGMR, TL, KT, YW, CSH, TJJJ, CN, KC, RGS, CC, SRD, BLH, RJS, DJG, and MDL contributed materials or analysis tools. EGMR, DJG, and MDL interpreted results. BLH, RJS, $D J G$, and $M D L$ were involved in supervising the project. EGMR, DJG, and $M D L$ wrote and revised the manuscript. All authors read and approved the final manuscript.

\section{Funding}

The research was financially supported by the Ministry of Business, Innovation and Employment (MBIE; Wellington, New Zealand) and the Ministry of Primary Industries (MPI; Wellington, New Zealand), who independently co-funded the work through the Endeavour Fund (LICX1802) and (now historical) Primary 
Growth Partnership research programs, respectively. EGMR is supported by a MPI Postgraduate Scholarship (Wellington, New Zealand) and an Al Rae Centre scholarship (Hamilton, New Zealand). External funders had no role in the analysis or interpretation of the data, or in writing the manuscript.

\section{Availability of data and materials}

A subset of whole-genome sequences used for imputation of the genotypes presented in this paper have been deposited in the SRA database [60]. Additional data is available on reasonable request with the permission of Livestock Improvement Corporation, contingent on the execution of an appropriate transfer agreement.

\section{Declarations}

\section{Ethics approval and consent to participate}

All animal experiments were conducted in strict accordance with the rules and guidelines outlined in the New Zealand Animal Welfare Act 1999. The majority of genotype and phenotype data were generated as part of routine commercial activities outside the scope of that requiring formal committee assessment (as defined by the above guidelines).

\section{Consent for publication}

\section{Not applicable.}

\section{Competing interests}

TL, YW, KT, CSH, TJJJ, CN, KC, RGS, CC, SRD, BLH, RJS, MDL are employees of Livestock Improvement Corporation (LIC; Hamilton, New Zealand), a commercial provider of bovine germplasm. Livestock Improvement Corporation is the applicant for several patent applications related to some of the mutations detailed in this article, with EGMR and MDL named inventors on these applications. Specifically, these filed patents relate to genetic testing applications of mutations impacting the DOCK8 (768802), IL4R (768803), KIAA0556 (768804), ITGAL (777216) LRCH4 (768805), and RBM34 (768806) genes. All other authors declare that they have no competing interests.

\section{Author details}

${ }^{1}$ Massey University, Palmerston North, New Zealand. ${ }^{2}$ Livestock Improvement Corporation, Hamilton, New Zealand.

Received: 30 Auqust 2021 Accepted: 21 December 2021 Published online: 24 January 2022

\section{References}

1. Charlier C, Agerholm JS, Coppieters W, Karlskov-Mortensen P, Li W, de Jong $\mathrm{G}$, et al. A deletion in the bovine FANCl gene compromises fertility by causing fetal death and brachyspina. PLoS One. 2012;7:e43085.

2. Littlejohn MD, Henty KM, Tiplady K, Johnson T, Harland C, Lopdell T, et al. Functionally reciprocal mutations of the prolactin signalling pathway define hairy and slick cattle. Nat Commun. 2014;5:5861.

3. Bourneuf $E$, Otz P, Pausch H, Jagannathan V, Michot P, Grohs C, et al. Rapid discovery of de novo deleterious mutations in cattle enhances the value of livestock as model species. Sci Rep. 2017;7:11466.

4. VanRaden PM, Olson KM, Null DJ, Hutchison JL. Harmful recessive effects on fertility detected by absence of homozygous haplotypes. J Dairy Sci. 2011;94:6153-61.

5. Charlier C, Li W, Harland C, Littlejohn M, Coppieters W, Creagh F, et al. NGS-based reverse genetic screen for common embryonic lethal mutations compromising fertility in livestock. Genome Res. 2016;26:1333-41.

6. Michot P, Chahory S, Marete A, Grohs C, Dagios D, Donzel E, et al. A reverse genetic approach identifies an ancestral frameshift mutation in $R P 1$ causing recessive progressive retinal degeneration in European cattle breeds. Genet Sel Evol. 2016:48:56.

7. Zhu Z, Bakshi A, Vinkhuyzen AAE, Hemani G, Lee SH, Nolte IM, et al. Dominance genetic variation contributes little to the missing heritability for human complex traits. Am J Hum Genet. 2015;96:377-85.

8. Bolormaa S, Pryce JE, Zhang Y, Reverter A, Barendse W, Hayes BJ, et al. Non-additive genetic variation in growth, carcass and fertility traits of beef cattle. Genet Sel Evol. 2015:47:26.
9. Sun C, VanRaden PM, Cole JB, O'Connell JR. Improvement of prediction ability for genomic selection of dairy cattle by including dominance effects. PLoS One. 2014;9:e103934.

10. Aliloo H, Pryce JE, González-Recio O, Cocks BG, Hayes BJ. Accounting for dominance to improve genomic evaluations of dairy cows for fertility and milk production traits. Genet Sel Evol. 2016:48:8.

11. Jiang J, Ma L, Prakapenka D, VanRaden PM, Cole JB, Da Y. A large-scale genome-wide association study in U.S. Holstein cattle. Front Genet. 2019;10:412.

12. Reynolds EGM, Neeley C, Lopdell TJ, Keehan M, Dittmer K, Harland CS, et al. Non-additive association analysis using proxy phenotypes identifies novel cattle syndromes. Nat Genet. 2021;53:949-54.

13. Grisart B, Coppieters W, Farnir F, Karim L, Ford C, Berzi P, et al. Positional candidate cloning of a QTL in dairy cattle: identification of a missense mutation in the bovine DGAT1 gene with major effect on milk yield and composition. Genome Res. 2002;12:222-31.

14. Blott S, Kim JJ, Moisio S, Schmidt-Küntzel A, Cornet A, Berzi P, et al. Molecular dissection of a quantitative trait locus: a phenylalanine-totyrosine substitution in the transmembrane domain of the bovine growth hormone receptor is associated with a major effect on milk yield and composition. Genetics. 2003;163:253-66.

15. Cohen-Zinder M, Seroussi E, Larkin DM, Loor JJ, Everts-Van Der Wind A, Lee $J H$, et al. Identification of a missense mutation in the bovine ABCG2 gene with a major effect on the QTL on chromosome 6 affecting milk yield and composition in Holstein cattle. Genome Res. 2005;15:936-44.

16. Littlejohn MD, Tiplady K, Lopdell T, Law TA, Scott A, Harland C, et al. Expression variants of the lipogenic AGPAT6 gene affect diverse milk composition phenotypes in Bos taurus. PLoS One. 2014;9:85757.

17. Littlejohn MD, Tiplady K, Fink TA, Lehnert K, Lopdell T, Johnson T, et al. Sequence-based association analysis reveals an MGST1 eQTL with pleiotropic effects on bovine milk composition. Sci Rep. 2016:6:25376.

18. Bauman DE, Currie BW. Partitioning of nutrients during pregnancy and lactation: a review of mechanisms involving homeostasis and homeorhesis. J Dairy Sci. 1980;63:1514-29.

19. Garrick DJ, Taylor JF, Fernando RL. Deregressing estimated breeding values and weighting information for genomic regression analyses. Genet Sel Evol. 2009;41:55

20. Li H. Aligning sequence reads, clone sequences and assembly contigs with BWA-MEM. 2013. https://arxiv.org/pdf/1303.3997.pdf.

21. Depristo MA, Banks E, Poplin R, Garimella KV, Maguire JR, Hartl C, et al. A framework for variation discovery and genotyping using next-generation DNA sequencing data. Nat Genet. 2011:43:491-8.

22. Browning BL, Zhou Y, Browning SR. A one-penny imputed genome from next-generation reference panels. Am J Hum Genet. 2018;103:338-48.

23. Wang Y. Evaluating the accuracy of imputed whole-genome sequence data in admixed dairy cattle. In: Proceedings of the 6th international conference of quantitative genetics: 2-3 and 12-13 November 2020; Brisbane (virtual). 2020:427

24. Druet T, Georges M. LINKPHASE3: an improved pedigree-based phasing algorithm robust to genotyping and map errors. Bioinformatics. 2015:31:1677-9.

25. Pausch H, MacLeod IM, Fries R, Emmerling R, Bowman PJ, Daetwyler HD, et al. Evaluation of the accuracy of imputed sequence variant genotypes and their utility for causal variant detection in cattle. Genet Sel Evol. 2017:49:24.

26. Yang J, Lee $\mathrm{SH}$, Goddard ME, Visscher PM. GCTA: a tool for genome-wide complex trait analysis. Am J Hum Genet. 2011;88:76-82.

27. Fernando RL, Garrick D. Bayesian methods applied to GWAS. In: Gondro C, van der Werf J, Hayes B, editors. Genome-wide association studies and genomic prediction Methods in Molecular Biology (Methods and Protocols). Totowa: Humana Press; 2013. p. 237-74.

28. Geweke JF, et al. Evaluating the accuracy of sampling-based approaches to the calculation of posterior moments. Staff report, Federal Reserve Bank of Minneapolis. 1991;148.

29. Bernal Rubio YL, Gualdrón Duarte JL, Bates RO, Ernst CW, Nonneman $D$, Rohrer $G A$, et al. Meta-analysis of genome-wide association from genomic prediction models. Anim Genet. 2015;47:36-48.

30. Storey JD, Tibshirani R. Statistical significance for genomewide studies. Proc Natl Acad Sci USA. 2003;100:9440-5.

31. Storey JD, Bass AJ, Dabney A, Robinson D. qvalue: Q-value estimation for false discovery rate control. R package version 2.18. 2019. 
32. Yang J, Ferreira T, Morris AP, Medland SE, GIANT Consortium, DIAGRAM Consortium, et al. Conditional and joint multiple-SNP analysis of GWAS summary statistics identifies additional variants influencing complex traits. Nat Genet. 2012;44:369-75.

33. McLaren W, Gil L, Hunt SE, Riat HS, Ritchie GRS, Thormann A, et al. The ensembl variant effect predictor. Genome Biol. 2016;17:122.

34. Ng PC, Henikoff S. SIFT: predicting amino acid changes that affect protein function. Nucleic Acids Res. 2003;31:3812-4.

35. Robinson JT, Thorvaldsdóttir H, Winckler W, Guttman M, Lander ES, Getz $\mathrm{G}$, et al. Integrative genomics viewer. Nat Biotechnol. 2011;29:24-6.

36. Jiang J, Shen B, O'Connell JR, VanRaden PM, Cole JB, Ma L. Dissection of additive, dominance, and imprinting effects for production and reproduction traits in Holstein cattle. BMC Genomics. 2017;18:425.

37. Lopdell TJ, Tiplady K, Couldrey C, Johnson TJJ, Keehan M, Davis SR, et al. Multiple QTL underlie milk phenotypes at the CSF2RB locus. Genet Sel Evol. 2019;51:3.

38. Lopdell TJ, Tiplady K, Struchalin M, Johnson TJJ, Keehan M, Sherlock R, et al. DNA and RNA-sequence based GWAS highlights membrane-transport genes as key modulators of milk lactose content. BMC Genomics. 2017; 18:968.

39. Caroli AM, Chessa S, Erhardt GJ. Invited review: Milk protein polymorphisms in cattle: effect on animal breeding and human nutrition. J Dairy Sci. 2009;92:5335-52

40. Wei WH, Hemani G, Haley CS. Detecting epistasis in human complex traits. Nat Rev Genet. 2014;15:722-33.

41. Visscher PM, Wray NR, Zhang Q, Sklar P, McCarthy MI, Brown MA, et al. 10 years of GWAS discovery: biology, function, and translation. Am J Hum Genet. 2017;101:5-22.

42. Hiltpold M, Kadri NK, Janett F, Witschi U, Schmitz-Hsu F, Pausch H. Autosomal recessive loci contribute significantly to quantitative variation of male fertility in a dairy cattle population. BMC Genomics. 2021;22:225.

43. Kearney CJ, Randall KL, Oliaro J. DOCK8 regulates signal transduction events to control immunity. Cell Mol Immunol. 2017;14:406-11.

44. Engelhardt KR, McGhee S, Winkler S, Sassi A, Woellner C, Lopez-herrera $G$, et al. Large deletions and point mutations involving the dedicator of cytokinesis (DOCK8) in the autosomal recessive form of the hyper-lgE syndrome. J Allergy Clin Immunol. 2009;124:1289-302.e4.

45. Randall KL, Lambe T, Johnson AL, Treanor B, Kucharska E, Domaschenz $H$, et al. Dock8 mutations cripple B cell immunological synapses, germinal centers and long-lived antibody production. Nat Immunol. 2009;10:1283-91.

46. Lambe T, Crawford G, Johnson AL, Crockford TL, Bouriez-Jones T, Smyth AM, et al. DOCK8 is essential for T-cell survival and the maintenance of CD8 + T-cell memory. Eur J Immunol. 2011;41:3423-35.

47. Zhang Q, Dove CG, Hor JL, Murdock HM, Strauss-Albee DM, Garcia JA, et al. DOCK8 regulates lymphocyte shape integrity for skin antiviral immunity. J Exp Med. 2014;211:2549-66.

48. Janssen E, Tsitsikov E, Al-Herz W, Lefranc G, Megarbane A, Dasouki M, et al. Flow cytometry biomarkers distinguish DOCK8 deficiency from severe atopic dermatitis. Clin Immunol. 2014;150:220-4.

49. Shirakawa T, Deichmann KA, Izuhara K, Mao XQ, Adra CN, Hopkin JM. Atopy and asthma: Genetic variants of IL-4 and IL-13 signalling. Immunol Today. 2000;21:60-4.

50. Sanders AAWM, de Vrieze E, Alazami AM, Alzahrani F, Malarkey EB, Sorusch N, et al. KIAA0556 is a novel ciliary basal body component mutated in Joubert syndrome. Genome Biol. 2015;16:293.

51. Zhang J, Teh M, Kim J, Eva MM, Cayrol R, Meade R, et al. A loss-of-function mutation in the Integrin alpha L (Itgal) gene contributes to susceptibility to Salmonella enterica serovar Typhimurium infection in Collaborative Cross strain CC042. Infect Immun. 2019;88:e00656-e719.

52. Aloor JJ, Azzam KM, Guardiola JJ, Gowdy KM, Madenspacher JH, Gabor KA, et al. Leucine-rich repeats and calponin homology containing 4 (Lrch4) regulates the innate immune response. J Biol Chem. 2019;294:1997-2008.

53. Graham BH, Waymire KG, Cottrell B, Trounce IA, MacGregor GR, Wallace DC. A mouse model for mitochondrial myopathy and cardiomyopathy resulting from a deficiency in the heart/muscle isoform of the adenine nucleotide translocator. Nat Genet. 1997;16:226-34.

54. Kaukonen J, Juselius JK, Tiranti V, Kyttälä A, Zeviani M, Comi GP, et al. Role of adenine nucleotide translocator 1 in mtDNA maintenance. Science. 2000;289:782-5.
55. Palmieri L, Alberio S, Pisano I, Lodi T, Meznaric-Petrusa M, Zidar J, et al. Complete loss-of-function of the heart/muscle-specific adenine nucleotide translocator is associated with mitochondrial myopathy and cardiomyopathy. Hum Mol Genet. 2005;14:3079-88.

56. Wang X, Ping C, Tan P, Sun C, Liu G, Liu T, et al. hnRNPLL controls pluripotency exit of embryonic stem cells by modulating alternative splicing of Tbx3 and Bptf. EMBO J. 2021;40:e104729.

57. Raven LA, Cocks BG, Kemper KE, Chamberlain AJ, Vander Jagt CJ, Goddard ME, et al. Targeted imputation of sequence variants and gene expression profiling identifies twelve candidate genes associated with lactation volume, composition and calving interval in dairy cattle. Mamm Genome. 2016;27:81-97.

58. Karim L, Takeda H, Lin L, Druet T, Arias JA, Baurain D, et al. Variants modulating the expression of a chromosome domain encompassing PLAG1 influence bovine stature. Nat Genet. 2011;43:405-13.

59. Fink T, Tiplady K, Lopdell T, Johnson T, Snell RG, Spelman RJ, et al. Functional confirmation of PLAG1 as the candidate causative gene underlying major pleiotropic effects on body weight and milk characteristics. Sci Rep. 2017;7:44793.

60. Livestock Improvement Corporation. PRJNA656361 Cattle whole genome sequences. 2021. https://www.ncbi.nlm.nih.gov/bioproject/PRJNA 656361. Accessed 25 Nov 2021.

\section{Publisher's Note}

Springer Nature remains neutral with regard to jurisdictional claims in published maps and institutional affiliations.

Ready to submit your research? Choose BMC and benefit from:

- fast, convenient online submission

- thorough peer review by experienced researchers in your field

- rapid publication on acceptance

- support for research data, including large and complex data types

- gold Open Access which fosters wider collaboration and increased citations

- maximum visibility for your research: over $100 \mathrm{M}$ website views per year

At BMC, research is always in progress.

Learn more biomedcentral.com/submissions 